\title{
The nature of $X(3900)$ and recognition of open charm effects
}

\author{
Qian Wang* \\ Institute of High Energy Physics, Chinese Academy of Sciences, Beijing 100049, China \\ E-mail: wangqiandihep.ac.cn
}

\section{Gang Li}

Department of Physics, Qufu Normal University, Qufu, 273165, China

E-mail: ligang0314@tom.com

\section{Xiao Hai Liu}

Institute of High Energy Physics, Chinese Academy of Sciences, Beijing 100049, China

E-mail: xhliulihep.ac.cn

\section{Qiang Zhao}

Institute of High Energy Physics, Chinese Academy of Sciences, Beijing 100049, China and Theoretical Physics Center for Science Facilities, CAS, Beijing 100049, China

E-mail: zhaog@ihep.ac.cn

We identify open charm effects in a direct production process $e^{+} e^{-} \rightarrow J / \psi \pi^{0}$. A unique feature of this process is that the $D \bar{D}^{*}+$ c.c. threshold is located at a relatively isolated energy region, i.e. $\sim 3.876 \mathrm{GeV}$, which is far away from the well-established charmonia $\psi(3770)$ and $\psi(4040)$. Therefore, the cross section line-shape of this reaction provides an opportunity for singling out the open charm effects. A model-independent narrow enhancement between the thresholds of $D^{0} \bar{D}^{* 0}+$ c.c. and $D^{+} D^{*-}+$ c.c. is predicted. This study can also help understand the $X(3900)$ enhancement recently observed by the Belle and BaBar Collaboration in $e^{+} e^{-} \rightarrow D \bar{D}+$ c.c. We also show that the open charm effects play a crucial role for our understanding of the long-standing " $\rho \pi$ puzzle"

Sixth International Conference on Quarks and Nuclear Physics April 16-20, 2012

Ecole Polytechnique, Palaiseau, Paris

\footnotetext{
* Speaker.
} 


\section{Introduction}

The study of hadron spectroscopy covers a broad energy region from low to high energies, and provides important information about the dynamics of the strong interaction. The experimental progress during the past years has brought a lot of surprises to our community. Taking the charmonium sector as an example, a number of new resonance-like signals have been observed by the B-factories [1]. These observations have aroused great interests from both theory and experiment in understanding their nature and searching for signals for exotic hadrons (e.g. see Refs. [2, 3, 4, 5] for a recent review on these issues).

Although various theoretical prescriptions, such as hybrid charmonium, tetraquark, baryonium, and hadronic molecule, have been proposed in order to understand the underlying dynamics for the production and decay of these new charmonium-like "resonances", an obvious feature with those signals is that most of them are close to open charmed meson thresholds. A typical example is the $X(3872)$ which is located between the $D^{0} \bar{D}^{* 0}$ and $D^{+} D^{*-}$ thresholds. As a result, a molecular prescription has been broadly investigated in the literature. It makes the non-perturbative mechanisms arising from the open charm thresholds an attractive solution for some of those charmoniumlike states.

The Belle Collaboration [6] recently observe an enhancement $X(3900)$ in ISR $e^{+} e^{-} \rightarrow D \bar{D}+$ c.c. process. The interesting feature about this enhancement is that it is directly produced in the $e^{+} e^{-}$annihilation, thus, its quantum number should be $J^{P C}=1^{--}$. Meanwhile, one notices that below and above the $X(3900)$ there are two well established charmonium states, $\psi(3770)$ and $\psi(4040)$, which can be consistently accommodated into the charmonium spectrum as $\psi(1 D)$ and $\psi(3 S)$ states, respectively. In another word, the $X(3900)$ enhancement is located in a mass region where the quark model does not have a corresponding $c \bar{c}$ vector state.

Although such an enhancement was conjectured to be caused by the $D \bar{D}^{*}+$ c.c. open charm effects, other possibilities seem not to be eliminated. In Ref. [7], the $D \bar{D}^{*}+$ c.c. open charm effects are investigated and the results seem to support such an explanation without introducing any exotic components. However, it is not obvious to conclude such a scenario since it is also shown in Ref. [7] that the enhancement can also be fitted by a Breit-Wigner structure. In order to clarify the nature of the $X(3900)$, one should investigate other possible reflections of such a mechanism. In this proceeding, we present our recent works on identifying an open charm effect in $e^{+} e^{-}$annihilations [8] and $e^{+} e^{-} \rightarrow J / \psi \eta, J / \psi \pi^{0}$ and $\phi \eta_{c}$, where a significant model-independent enhancement at about $3.876 \mathrm{GeV}$ is predicted in the process $e^{+} e^{-} \rightarrow J / \psi \pi^{0}$ as a direct evidence for the $D \bar{D}^{*}+$ c.c. open charm effects.

As a dynamical mechanism, the open charm effects can explain the discrepancies between the experimental data and pQCD predictions for, e.g. $\psi(3770)$ non- $D \bar{D}$ decay [9] and helicity selection rule evading processes [10, 11, 12]. As a further evidence for the open charm effects, we also present our recent studies of the long-standing " $\rho \pi$ puzzle" in $J / \psi$ and $\psi^{\prime}$ decays in this proceeding [13].

\section{The recognition of the open charm effects in the process $e^{+} e^{-} \rightarrow J / \psi \pi^{0}$}

Based on the effective Lagrangians $[11,12,14,15,16]$ and vector meson dominance (VMD) 
model, the amplitudes of the diagrams as shown in Fig. 1 can be obtained and the details can be found in Ref. [8]. In the energy region that we are interested in, five vector charmonia, i.e. $J / \psi$, $\psi(3686), \psi(3770), \psi(4040)$ and $\psi(4160)$, are included as the dominant pole contributions.

As shown in Fig. 1, both neutral and charged $D$ meson loops can contribute to the $J / \psi \eta$ and $J / \psi \pi^{0}$ production channels. These two amplitudes have the same sign for the isospin conserved process, i.e. $J / \psi \eta$ mode, but opposite sign for the isospin violating $J / \psi \pi^{0}$ mode. If isospin symmetry is respected, i.e. $u$ and $d$ quark have the same mass or the charged and neutral $D$ mesons approximately have the same mass, these two $D$ meson loops would cancel each other in the isospin violating process. If not, the charmed meson loops will then give non-zero residual contribution as a manifestation of the strong isospin violation.

Figure 2(a) gives the cross section of $e^{+} e^{-} \rightarrow J / \psi \eta$ in terms of the c.m. energy. The parameter in the form factor is obtained by normalizing the cross section at $\psi(3686)$ to the experimental value. These vertical lines are different charmed meson pair thresholds. Although the neutral and charged charmed meson loops have a constructive sign, we cannot see a clear $D \bar{D}^{*}$ open threshold effect in $e^{+} e^{-} \rightarrow J / \psi \eta$ due to large contributions from other processes. Namely, these threshold effects are submerged by the dominant resonance contributions.

Although the cross sections of these channels are sensitive to the cut-off parameter $\alpha$, the ratios between them are not. As a result, with the cut-off parameter $\alpha$ fixed in $e^{+} e^{-} \rightarrow J / \psi \eta$, we can predict the cross section of $e^{+} e^{-} \rightarrow J / \psi \pi^{0}$ as shown in Fig. 2(b). Since the charged and neutral $D$ meson loops have opposite signs in the $J / \psi \pi^{0}$ production mode, the resonance contributions are canceled largely. So we can see a much clearer enhancement between the $D^{0} \bar{D}^{* 0}$ and $D^{ \pm} D^{* \mp}$ thresholds, i.e. $\sim 3.876 \mathrm{GeV}$. Confirmation of such a phenomenon would suggest that the $\mathrm{X}(3900)$ can originate from the $D \bar{D}^{*}$ open threshold effects instead of a new charmonium state. Nevertheless, observation of such an enhancement in $e^{+} e^{-} \rightarrow J / \psi \pi^{0}$ should be a direct evidence for such a non-perturbative dynamic mechanisms.

As a byproduct, we also predict the cross section of $e^{+} e^{-} \rightarrow \phi \eta_{c}$ as shown in Fig. 2(c). There is also a kink structure at the $D_{s} D_{s}^{*}$ threshold. Unfortunately, since it is near $\psi(4040)$, their interference make this open charm effect quite insignificant.

\section{A possible explanation of the " $\rho \pi$ puzzle"}

The " $\rho \pi$ puzzle" in the literature is related to the power law suppression due to the pQCD helicity selection rule (HSR). For $J / \psi$ and $\psi^{\prime} \rightarrow V P$, it was shown that these two decays should be strongly suppressed at leading twist [17, 18]. Meanwhile, for the inclusive decays of $J / \psi$ and $\psi^{\prime}$ into light hadrons via the $c \bar{c}$ annihilation, it can be related to their leptonic decays since both processes probe the charmonium wavefunctions at the origins, i.e.

$$
R \equiv \frac{B R\left(\psi^{\prime} \rightarrow \text { hadrons }\right)}{B R(J / \psi \rightarrow \text { hadrons })} \simeq \frac{B R\left(\psi^{\prime} \rightarrow e^{+} e^{-}\right)}{B R\left(J / \psi \rightarrow e^{+} e^{-}\right)} \simeq 0.13
$$

which is the so called " $12 \%$ rule" [17]. It is interesting to observe that many exclusive processes respect this relation quite well, while some processes deviate from this rate drastically. In particular, the $\rho \pi$ and $K^{*} \bar{K}+$ c.c. channels have significant deviations from the expectations of both the pQCD HSR and " $12 \%$ rule". This originates the " $\rho \pi$ puzzle" in the literature and has attracted a lot of attention from both theory and experiment. 


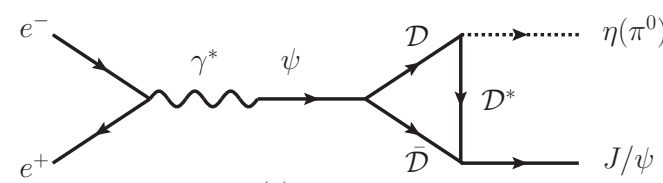

(a)

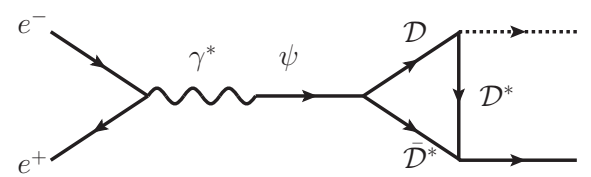

$(c)$

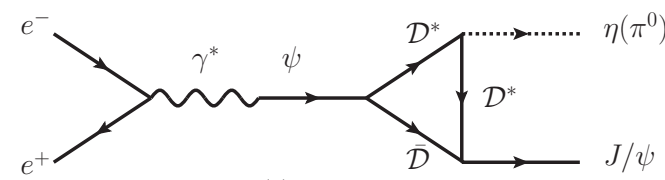

(e)

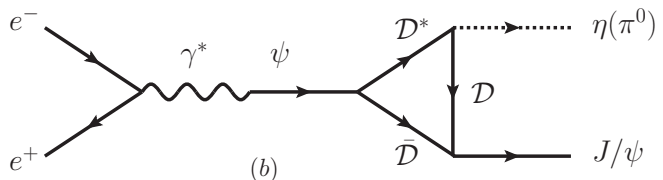

$(b)$

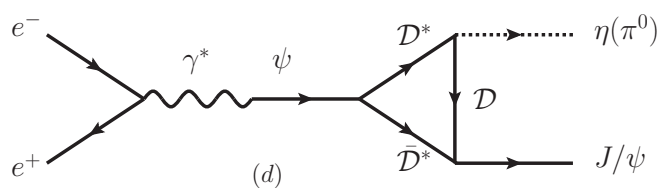

$(d)$

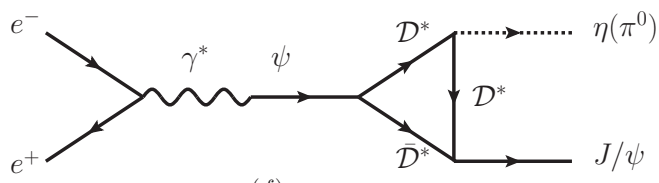

$(f)$

Figure 1: Schematic diagrams for $e^{+} e^{-} \rightarrow J / \psi \eta\left(\pi^{0}\right)$ via charmed $D\left(D^{*}\right)$ meson loops. The diagrams for the $\phi \eta_{c}$ mode are similar.
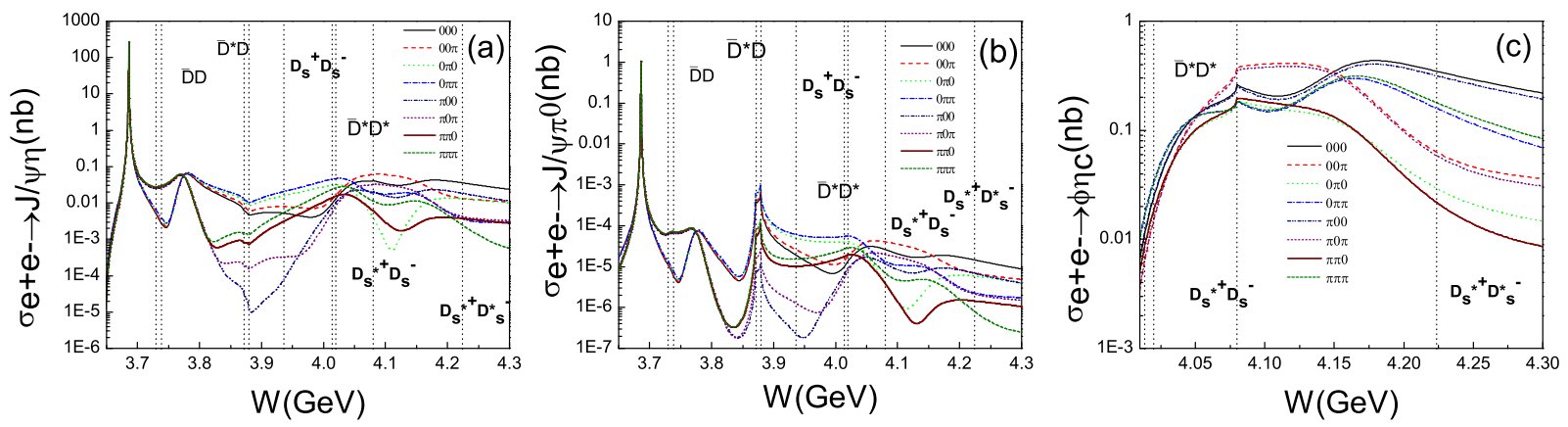

Figure 2: The predicted cross sections for $e^{+} e^{-} \rightarrow J / \psi \eta, J / \psi \pi^{0}, \phi \eta_{c}$ in terms of the c.m. energy $W$ with the cutoff parameter $\alpha=1.57$ are shown respectively. The cross sections with different phases, i.e. $(\theta, \beta, \phi)=(0,0,0),(0,0, \pi),(0, \pi, 0),(0, \pi, \pi),(\pi, 0,0),(\pi, 0, \pi),(\pi, \pi, 0),(\pi, \pi, \pi)$, are presented and denoted by different curves. The vertical lines labels the open charm thresholds.

In our analysis, we put constraints on the electromagnetic (EM) contribution, short-distance contribution from the $c \bar{c}$ annihilation at the wavefunction origin, and long-distance contribution from the open charm threshold effects on these two decays. We show that interferences among these amplitudes, in particular, the destructive interferences between the short-distance and long-distance strong amplitudes play a key role to cause the significant deviations from the pQCD expected " $12 \%$ rule". Since the mass of $\psi^{\prime}$ is closer to the open $D \bar{D}$, it would experience much more significant influences from the long-distance open charm threshold effects than the $J / \psi$. As a consequence, an overall suppression of the strong transition amplitudes for $\psi^{\prime} \rightarrow V P$ can be recognized. Such a phenomenon has also been realized in some early analyses though the dynamic reason for such a 
Table 1: Theoretical results for the branching ratios of $J / \psi\left(\psi^{\prime}\right) \rightarrow V P$ calculated in our model. The experimental data are from PDG2010 [19].

\begin{tabular}{|c|c|c|c|c|c|c|}
\hline$B R(J / \psi \rightarrow V P)$ & EM & short-distance & long-distance & strong & total & exp. \\
\hline$\rho \eta$ & $1.81 \times 10^{-4}$ & 0 & $2.34 \times 10^{-12}$ & $2.34 \times 10^{-12}$ & $1.81 \times 10^{-4}$ & $(1.93 \pm 0.23) \times 10^{-4}$ \\
\hline$\rho \eta^{\prime}$ & $1.37 \times 10^{-4}$ & 0 & $2.21 \times 10^{-12}$ & $2.21 \times 10^{-12}$ & $1.37 \times 10^{-4}$ & $(1.05 \pm 0.18) \times 10^{-4}$ \\
\hline$\omega \pi^{0}$ & $3.1 \times 10^{-4}$ & 0 & $2.38 \times 10^{-12}$ & $2.38 \times 10^{-12}$ & $3.10 \times 10^{-4}$ & $(4.5 \pm 0.5) \times 10^{-4}$ \\
\hline$\phi \pi^{0}$ & $9.52 \times 10^{-7}$ & 0 & 0 & 0 & $9.52 \times 10^{-7}$ & $<6.4 \times 10^{-6}$ \\
\hline$\rho^{0} \pi^{0}$ & $4.44 \times 10^{-5}$ & $4.85 \times 10^{-3}$ & $2.24 \times 10^{-7}$ & $4.89 \times 10^{-3}$ & $5.87 \times 10^{-3}$ & $(5.6 \pm 0.7) \times 10^{-3}$ \\
\hline$\rho \pi$ & $1.06 \times 10^{-4}$ & $1.45 \times 10^{-2}$ & $6.71 \times 10^{-7}$ & $1.47 \times 10^{-2}$ & $1.73 \times 10^{-2}$ & $(1.69 \pm 0.15) \times 10^{-2}$ \\
\hline$K^{*+} K^{-}+c . c$. & $6.97 \times 10^{-5}$ & $4.69 \times 10^{-3}$ & $3.14 \times 10^{-7}$ & $4.74 \times 10^{-3}$ & $5.96 \times 10^{-3}$ & $(5.12 \pm 0.3) \times 10^{-3}$ \\
\hline$K^{* 0} \bar{K}^{0}+c . c$. & $1.59 \times 10^{-4}$ & $4.68 \times 10^{-3}$ & $3.11 \times 10^{-7}$ & $4.73 \times 10^{-3}$ & $3.16 \times 10^{-3}$ & $(4.39 \pm 0.31) \times 10^{-3}$ \\
\hline$\omega \eta$ & $1.4 \times 10^{-5}$ & $1.76 \times 10^{-3}$ & $1.50 \times 10^{-7}$ & $1.78 \times 10^{-3}$ & $2.11 \times 10^{-3}$ & $(1.74 \pm 0.20) \times 10^{-3}$ \\
\hline$\omega \eta^{\prime}$ & $1.4 \times 10^{-5}$ & $9.91 \times 10^{-5}$ & $5.42 \times 10^{-8}$ & $1.02 \times 10^{-4}$ & $1.92 \times 10^{-4}$ & $(1.82 \pm 0.21) \times 10^{-4}$ \\
\hline$\phi \eta$ & $2.35 \times 10^{-5}$ & $6.70 \times 10^{-4}$ & $3.22 \times 10^{-8}$ & $6.76 \times 10^{-4}$ & $9.52 \times 10^{-4}$ & $(7.5 \pm 0.8) \times 10^{-4}$ \\
\hline$\phi \eta^{\prime}$ & $2.10 \times 10^{-5}$ & $2.07 \times 10^{-4}$ & $6.45 \times 10^{-8}$ & $2.12 \times 10^{-4}$ & $9.93 \times 10^{-5}$ & $(4.0 \pm 0.7) \times 10^{-4}$ \\
\hline \multicolumn{7}{|l|}{$\overline{B R R\left(\psi^{\prime} \rightarrow V P\right)}$} \\
\hline$\rho \eta$ & $1.42 \times 10^{-5}$ & 0 & $4.13 \times 10^{-7}$ & $4.13 \times 10^{-7}$ & $1.94 \times 10^{-5}$ & $(2.2 \pm 0.6) \times 10^{-5}$ \\
\hline$\rho \eta^{\prime}$ & $1.04 \times 10^{-5}$ & 0 & $3.89 \times 10^{-7}$ & $3.89 \times 10^{-7}$ & $1.48 \times 10^{-5}$ & $\left(1.9_{-1.2}^{+1.7}\right) \times 10^{-5}$ \\
\hline$\omega \pi^{0}$ & $2.98 \times 10^{-5}$ & 0 & $4.25 \times 10^{-7}$ & $4.25 \times 10^{-7}$ & $3.73 \times 10^{-5}$ & $(2.1 \pm 0.6) \times 10^{-5}$ \\
\hline$\phi \pi^{0}$ & $9.78 \times 10^{-8}$ & 0 & 0 & 0 & $9.78 \times 10^{-8}$ & $<4.0 \times 10^{-6}$ \\
\hline$\rho^{0} \pi^{0}$ & $4.36 \times 10^{-6}$ & $5.81 \times 10^{-4}$ & $7.85 \times 10^{-4}$ & $2.12 \times 10^{-5}$ & $9.72 \times 10^{-6}$ & $* * *$ \\
\hline$\rho \pi$ & $1.02 \times 10^{-5}$ & $1.74 \times 10^{-3}$ & $2.36 \times 10^{-3}$ & $6.36 \times 10^{-5}$ & $3.20 \times 10^{-5}$ & $(3.2 \pm 1.2) \times 10^{-5}$ \\
\hline$K^{*+} K^{-}+c . c$. & $7.03 \times 10^{-6}$ & $9.81 \times 10^{-4}$ & $1.33 \times 10^{-3}$ & $3.64 \times 10^{-5}$ & $1.70 \times 10^{-5}$ & $\left(1.7_{-0.7}^{+0.8}\right) \times 10^{-5}$ \\
\hline$K^{* 0} \bar{K}^{0}+c . c$. & $1.61 \times 10^{-5}$ & $9.81 \times 10^{-4}$ & $1.39 \times 10^{-3}$ & $4.61 \times 10^{-5}$ & $1.09 \times 10^{-4}$ & $(1.09 \pm 0.20) \times 10^{-4}$ \\
\hline$\omega \eta$ & $1.10 \times 10^{-6}$ & $3.24 \times 10^{-4}$ & $5.57 \times 10^{-4}$ & $3.52 \times 10^{-5}$ & $2.48 \times 10^{-5}$ & $<1.1 \times 10^{-5}$ \\
\hline$\omega \eta^{\prime}$ & $1.12 \times 10^{-6}$ & $6.23 \times 10^{-5}$ & $2.31 \times 10^{-4}$ & $5.43 \times 10^{-5}$ & $4.01 \times 10^{-5}$ & $\left(3.2_{-2.1}^{+2.5}\right) \times 10^{-5}$ \\
\hline$\phi \eta$ & $2.26 \times 10^{-6}$ & $1.55 \times 10^{-4}$ & $1.73 \times 10^{-4}$ & $1.92 \times 10^{-6}$ & $2.25 \times 10^{-6}$ & $\left(2.8_{-0.8}^{+1.0}\right) \times 10^{-5}$ \\
\hline$\phi \eta^{\prime}$ & $2.22 \times 10^{-6}$ & $1.85 \times 10^{-4}$ & $3.99 \times 10^{-4}$ & $4.33 \times 10^{-5}$ & $6.42 \times 10^{-5}$ & $(3.1 \pm 1.6) \times 10^{-5}$ \\
\hline
\end{tabular}

suppression were not clarified [20, 21, 22, 23]. In Ref. [13] the role played by the open charmed meson loops are quantified.

Our final results are shown in Table 1 with different transitions given explicitly. The charmed meson loop plays a significant role in $\psi^{\prime}$ decays, since it closes to the $D \bar{D}$ threshold. In contrast, it is easy to understand its negligible influences in $J / \psi$ decays. Meanwhile, the short-distance parts of these two decays respect the $12 \%$ rule. The destructive interferences between the short-distance and long-distance part gives the suppression of the strong transition amplitudes in the $\psi^{\prime}$ decays, which then become comparable with the EM contribution.

\section{Summary}

In summary, we present our recent results on the study of the open charm effects in $e^{+} e^{-} \rightarrow$ $J / \psi \eta, J / \psi \pi^{0}, \phi \eta_{c}$. We identify a model-independent enhancement at about $3.876 \mathrm{GeV}$ in $e^{+} e^{-} \rightarrow J / \psi \pi^{0}$ due to the isospin violating contributions via the open charm effects. Such a prediction, if confirmed, would suggest that the $X(3900)$ may be due to the $D \bar{D}^{*}+$ c.c. open threshold effects instead of a new charmonium state. Such a mechanism can be directly examined in high statistics measurement of $e^{+} e^{-} \rightarrow J / \psi \pi^{0}$. Confirmation of this mechanism could be essential for our understanding of the long-standing " $\rho \pi$ puzzle". 


\section{Acknowledgment}

This work is supported, in part, by National Natural Science Foundation of China (Grant Nos. 11035006 and 10947007), Chinese Academy of Sciences (KJCX2-EW-N01), Ministry of Science and Technology of China (2009CB825200), and the Natural Science Foundation of Shandong Province (Grant No. ZR2010AM011) and the Scientific Research Starting Foundation of Qufu Normal University.

\section{References}

[1] S. L. Olsen, arXiv:0909.2713 [hep-ex].

[2] M. B. Voloshin, Prog. Part. Nucl. Phys. 61, 455 (2008) [arXiv:0711.4556 [hep-ph]].

[3] E. Eichten, S. Godfrey, H. Mahlke and J. L. Rosner, Rev. Mod. Phys. 80, 1161 (2008) [arXiv:hep-ph/0701208].

[4] N. Brambilla, S. Eidelman, B. K. Heltsley, R. Vogt, G. T. Bodwin, E. Eichten, A. D. Frawley and A. B. Meyer et al., Eur. Phys. J. C 71, 1534 (2011) [arXiv:1010.5827 [hep-ph]].

[5] N. Drenska, R. Faccini, F. Piccinini, A. Polosa, F. Renga, C. Sabelli, Riv. Nuovo Cim. 033, 633-712 (2010). [arXiv:1006.2741 [hep-ph]].

[6] G. Pakhlova et al. [Belle Collaboration], Phys. Rev. D 77, 011103 (2008) [arXiv:0708.0082 [hep-ex]].

[7] Y. J. Zhang and Q. Zhao, Phys. Rev. D 81, 034011 (2010) [arXiv:0911.5651 [hep-ph]].

[8] Q. Wang, X. -H. Liu and Q. Zhao, Phys. Rev. D 84, 014007 (2011) [arXiv:1103.1095 [hep-ph]].

[9] Y. J. Zhang, G. Li and Q. Zhao, Phys. Rev. Lett. 102, 172001 (2009) [arXiv:0902.1300 [hep-ph]].

[10] Q. Wang, X. -H. Liu and Q. Zhao, Phys. Lett. B 711, 364 (2012) [arXiv:1202.3026 [hep-ph]].

[11] X. -H. Liu and Q. Zhao, J. Phys. G G 38, 035007 (2011) [arXiv:1004.0496 [hep-ph]].

[12] X. -H. Liu and Q. Zhao, Phys. Rev. D 81, 014017 (2010) [arXiv:0912.1508 [hep-ph]].

[13] Q. Wang, G. Li and Q. Zhao, Phys. Rev. D 85, 074015 (2012) [arXiv:1201.1681 [hep-ph]].

[14] Q. Wang, X. H. Liu and Q. Zhao, arXiv:1010.1343 [hep-ph].

[15] H. Y. Cheng, C. K. Chua and A. Soni, Phys. Rev. D 71, 014030 (2005) [arXiv:hep-ph/0409317].

[16] R. Casalbuoni, A. Deandrea, N. Di Bartolomeo, R. Gatto, F. Feruglio and G. Nardulli, Phys. Rept. 281, 145 (1997) [arXiv:hep-ph/9605342].

[17] S. J. Brodsky, G. P. Lepage, Phys. Rev. D24, 2848 (1981).

[18] V. L. Chernyak, A. R. Zhitnitsky, Nucl. Phys. B201, 492 (1982).

[19] K. Nakamura et al. [Particle Data Group], J. Phys. G 37, 075021 (2010).

[20] A. Seiden, H. F. W. Sadrozinski, H. E. Haber, Phys. Rev. D38, 824 (1988).

[21] M. Suzuki, Phys. Rev. D63, 054021 (2001).

[22] G. Li, Q. Zhao, C. -H. Chang, J. Phys. G 35, 055002 (2008). [hep-ph/0701020].

[23] Q. Zhao, G. Li, C. -H. Chang, Phys. Lett. B645, 173-179 (2007). [hep-ph/0610223]. 